\title{
Lagrangian formalism and retarded classical electrodynamics
}

\author{
$X$. Jaen, J. Llosa, and A. Molina \\ Grup de Relativitat, Societat Catalana de Fisica (I.E.C.) and Departament Fisica Fonamental, Universitat \\ de Barcelona, Diagonal 647, E-08028 Barcelona, Spain
}

(Received 28 June 1988; accepted for publication 2 February 1989)

Unlike the $1 / c^{2}$ approximation, where classical electrodynamics is described by the Darwin

Lagrangian, here there is no Lagrangian to describe retarded (resp., advanced) classical

electrodynamics up to $1 / c^{3}$ for two-point charges with different masses.

\section{INTRODUCTION}

The noninteraction theorem of Currie et al. ${ }^{1}$ and its further generalizations $\mathrm{s}^{2,3}$ established that if position coordinates are to be taken as canonical, then there is no Poincaré invariant Hamiltonian system of directly interacting particles other than the trivial case of free particles.

The original result was actually proven in the instant form of Hamiltonian relativistic mechanics ${ }^{4}$ and has only recently been extended to the other two Dirac approaches to relativistic Hamiltonian dynamics, namely, the front- and point-form approaches ${ }^{5}$ and, also, to more general approaches. ${ }^{6,7}$

By means of a Legendre transformation, that negative result can be translated into its Lagrangian counterpart, namely, the nonexistence of Poincare invariant Lagrangian systems of directly interacting particles, apart from the above-mentioned case of free particles. (Note that here, the "Poincaré invariant Lagrangian system" only means that the Euler-Lagrange equations are Poincare invariant; it does not imply the Poincaré invariance of the Lagrangian function.)

However, this result does not exclude the existence of Lagrangian (resp., Hamiltonian) systems that are Poincaré invariant up to terms $1 / c^{2}$, that is, modulo $1 / c^{3}$. This case encompasses several well-known Lagrangians, e.g., Darwin, ${ }^{8}$ Einstein et al., ${ }^{9}$ Bopp, ${ }^{10}$ and Breit ${ }^{11}$ for classical spin charges. Going further into this approach, Martin and San $\mathrm{z}^{12}$ proved that there exist nontrivial Lagrangian systems of directly interacting particles that are Poincaré invariant up to $1 / c^{n}$, but only if $n<6$.

In a later paper, ${ }^{13}$ Martin and Sanz derive the most general form of a Lagrangian function such that (i) it is invariant under the Aristotle group (i.e., space rotations and space and time translations), (ii) it admits a Newtonian limit, (iii) it is separable, and (iv) it yields a system of equations of motion that is Poincaré invariant up to $1 / c^{3}$. Martin and Sanz $z^{13}$ also obtain some conditions to be fulfilled by the $1 / c^{4}$ part of the Lagrangian in order to guarantee the Poincare invariance of equations of motion up to this order: They finally prove that the approximated Lagrangians derived for systems of particles interacting through a classical field are not Poincaré invariant up to $1 / c^{4}$.

Reference 13 agrees with the well-known fact that although classical electrodynamics of point charges is described up to $1 / c^{4}$ terms by the Darwin Lagrangian and the equations of motion are relativistic invariant up to this order, the same does not hold for the Golubenkov-Smorodin- skii Lagrangian, ${ }^{14}$ i.e., the Lagrangian one would obtain from Fokker symmetric electrodynamics of two charges ${ }^{15}$ by a convenient $1 / c$ expansion. ${ }^{16,17}$

One point, which in our opinion is interesting, has not been considered in Ref. 13: Is there a Lagrangian system fulfilling conditions (i)_(iv) and describing retarded (resp., advanced) electrodynamics up to $1 / c^{3}$ ? [Note that this question would not make sense for symmetric electrodynamicshalf-retarded plus half-advanced-because time reversal invariance implies that only even powers of $1 / c$ occur in the Lagrangian.)

In the present paper we give a negative answer to the above question, taking as equations of motion those given by predictive relativistic retarded electrodynamics ${ }^{18}$ of two point charges up to $1 / c^{3}$.

\section{THE LAGRANGIAN}

The search is for a Lagrangian approximated up to $1 / c^{3}$. Meeting conditions (i)-(iv) of Sec. I is done as follows. ${ }^{13}$ First, an analytical dependence on the "small" parameter 1/ $c$ is assumed:

$$
L=\sum_{n=0}^{\infty} c^{-n} L^{(n)}\left(\mathbf{x}_{a}, \mathbf{v}_{b}, t\right) .
$$

Then, the Aristotle invariance condition (i) implies

$$
L^{(n)}\left(\mathbf{x}_{a}, \mathbf{v}_{b}, t\right)=L^{(n)}\left(r, s, q, v_{a}^{2}, v^{2}\right),
$$

where the Aristotle invariant variables

$$
\begin{aligned}
& r \equiv\left|\mathbf{x}_{1}-\mathbf{x}_{2}\right|, \quad s=\frac{1}{2}\left(\mathbf{x}_{1}-\mathbf{x}_{2}\right) \cdot\left(\mathbf{v}_{1}-\mathbf{v}_{2}\right), \\
& q \equiv \frac{1}{2}\left(\mathbf{x}_{1}-\mathbf{x}_{2}\right) \cdot\left(\mathbf{v}_{1}+\mathbf{v}_{2}\right), \quad v^{2} \equiv\left(\mathbf{v}_{1}-\mathbf{v}_{2}\right)^{2}
\end{aligned}
$$

have been introduced.

The Newtonian limit condition (ii) and the separability condition (iii) read, respectively, as

$$
L^{(0)}=\frac{1}{2} m_{1} v_{1}^{2}+\frac{1}{2} m_{2} v_{2}^{2}-V(r)
$$

and

$$
\lim _{r \rightarrow \infty} L=\sum_{a} m_{a} c^{2}\left\{1-\sqrt{1-v_{a}^{2} / c^{2}}\right\} .
$$

Equation (4) ensures that the Lagrangian (1) is nonsingular, at least for "small" values of $1 / c$; indeed,

$$
\frac{\partial^{2} L}{\partial v_{a}^{i} \partial v_{b}^{j}}=m_{a} \delta_{a b} \delta_{i j}+O\left(\frac{1}{c}\right),
$$

where the subscripts $a, b=1,2$.

Hence the Euler-Lagrange equations can be solved in the particle accelerations, thus yielding 


$$
\dot{\mathbf{v}}_{a}=\mu_{a}\left(\mathbf{x}_{b}, \mathbf{v}_{c}\right)=\sum_{n=0}^{\infty} \frac{1}{c^{n}} \mu_{a}^{(n)}\left(\mathbf{x}_{b}, \mathbf{v}_{c}\right),
$$

where the subscripts $a, b, c=1,2$.

The condition of Poincare invariance up to $1 / c^{3}$ [i.e., condition (iv) ] of the equations of motion (7) is then ensured by requiring $\mu_{a}\left(x_{b}, \mathbf{v}_{c}\right)$ to meet the Currie-Hill equations ${ }^{19}$ up to $1 / c^{3}$ :

$$
\begin{gathered}
\frac{v_{a}^{k}}{c^{2}}\left(x_{a j}-x_{b j}\right) \frac{\partial \mu_{b}^{i}}{\partial x_{a}^{k}}+\left[\frac{v_{a}^{k} v_{a j}}{c^{2}}+\frac{\mu_{a}^{k}}{c^{2}}\left(x_{a j}-x_{b j}\right)-\epsilon_{a} \delta_{j}^{k}\right] \\
\times \frac{\partial \mu_{b}^{i}}{\partial x_{a}^{k}}=\frac{1}{c^{2}}\left(2 \mu_{b}^{i} v_{b j}+v_{b}^{i} \mu_{b j}\right),
\end{gathered}
$$

where $\epsilon_{a}=1$. (Summation over repeated indices is understood.)

It has been proven elsewhere ${ }^{20}$ that these equations ( 8 ) are a consequence of requiring that the whole family of solutions of the equations of motion (7) are invariant under the action of the Poincare group on the space of the initial data $D=\left(\mathbf{x}_{10}, \mathbf{x}_{20} ; \mathbf{v}_{10}, \mathbf{v}_{20}\right)$. This is a sort of "world line condition" that basically states that provided that $\phi_{a}(t, D)$, where $a=1,2$, are the particle trajectories from some given initial data $D$ and $D^{\prime}$ are the transformed initial data (i.e., the initial data that would be "seen" from another inertial frame), then the world lines $\left[t, \phi_{a}(t, D)\right]$ transform into the world lines $\left[t^{\prime}, \phi_{a}\left(t^{\prime}, D^{\prime}\right)\right]$.

The conditions (2), (4), (5), and (8) constrain the Lagrangian to have the special form

$$
\begin{aligned}
L= & \frac{1}{2}\left(m_{1} v_{1}^{2}+m_{2} v_{2}^{2}\right)-V(r)+\frac{1}{c^{2}}\left\{\frac{1}{8}\left(m_{1} v_{1}^{4}+m_{2} v_{2}^{4}\right)\right. \\
& -V^{\prime}(r) \frac{q^{2}}{2 r}+\frac{V}{4}\left(v_{1}^{2}+v_{2}^{2}\right)+\frac{\alpha(r)}{4}\left(v_{1}^{2}-v_{2}^{2}\right) \\
& \left.+\alpha^{\prime}(r) \frac{s q}{r}+f\left(r, s, v^{2}\right)\right\}+\frac{1}{c^{3}}\left\{\frac{\gamma(r)}{4}\left(v_{1}^{2}-v_{2}^{2}\right)\right. \\
& \left.+\gamma^{\prime}(r) \frac{s q}{r}+g\left(r, s, v^{2}\right)\right\}+O\left(\frac{1}{c^{4}}\right),
\end{aligned}
$$

where a prime means derivative and $\alpha(r), \Gamma(r), f(r, s, y)$, and $g(r, s, y)$ are arbitrary functions subject to the limit conditions (for $r \rightarrow \infty$ )

$$
\begin{aligned}
\lim \alpha(r) & =\lim \left(\alpha^{\prime}(r) / r\right)=\lim f(r) \\
& =\lim \gamma(r)=\lim \left(\gamma^{\prime}(r) / r\right)=\lim g(r)=0 .
\end{aligned}
$$

[See Ref. 13, Sec. III, for the intermediate steps leading to Eq. (9); indeed, there Eq. (9) is labeled (3.13).]

\section{RETARDED (ADVANCED) ELECTRODYNAMICS OF TWO-POINT CHARGES}

The equations of motion for retarded (resp., advanced) electrodynamics of two directly interacting charges (without an intermediate field) are given by

$$
m_{a} \frac{d}{d t}\left(\gamma_{a} \mathbf{v}_{a}\right)=e_{a}\left[\mathbf{E}_{a^{\prime}}\left(\mathbf{x}_{a}, \epsilon\right)+\frac{\mathbf{v}_{a}}{c} \times \mathbf{B}_{a^{\prime}}\left(\mathbf{x}_{a}, \epsilon\right)\right],
$$

where the subscripts $a \neq a^{\prime}$ and $a, a^{\prime}=1,2$ and where $\gamma_{a}$ $=\sqrt{1-v_{a}^{2} / c^{2}}$. Here, $\mathbf{E}_{a^{\prime}}\left(\mathbf{x}_{a}, \epsilon\right)$ and $\mathbf{B}_{a^{\prime}}\left(\mathbf{x}_{a}, \epsilon\right)$ are the re- tarded $(\epsilon=-1)$ [resp., advanced $(\epsilon=+1)$ ] Lienard-Wiechert electric and magnetic fields associated (ad$j u n c t$ ) to the charge $a^{\prime} \neq a$. The trouble with Eq. (11) is that it is not an ordinary differential system because $\mathbf{E}_{\alpha^{\prime}}\left(\mathbf{x}_{a}, \epsilon\right)$ and $\mathbf{B}_{a^{\prime}}\left(\mathbf{x}_{a}, \epsilon\right)$ are only defined for null configurations of particle $a^{\prime}$ retarded (resp., advanced) relative to $a$, i.e., $\left(x_{1}^{\mu}-x_{2}^{\mu}\right) \times\left(x_{1_{\mu}}-x_{2_{\mu}}\right)=0$ and $\left(x_{a}^{0}-x_{a^{\prime}}^{0}\right) . \epsilon<0$.

Hence (11) is a difference-differential system and initial positions and velocities of particles do not determine a unique future evolution.

However, Eq. (11) can be taken as a boundary condition for solving the Currie-Hill equation (8) ${ }^{20}$ (The Currie-Hill equation (8) acts as a partial differential condition on the particle accelerations in order to ensure the Poincare invariance of the world lines.) Introducing the additional requirement that world lines depend analytically on the small parameter $1 / c$, the resulting equations of motion up to the order $1 / c^{3}$ are derived in Ref. 18 and read as

$$
\begin{aligned}
m_{a} \boldsymbol{\mu}_{a}= & \eta_{a}\left[-\frac{V^{\prime}}{r} \mathbf{r}+\frac{1}{c^{2}}\left\{-\mathbf{r}\left(\frac{V^{\prime}}{2 r}\left(v^{2}-2 v_{a}^{2}\right)\right.\right.\right. \\
& \left.\left.+\frac{1}{2 r}\left(\frac{V^{\prime}}{r}\right)^{\prime}\left(\mathbf{r} \cdot \mathbf{v}_{a^{\prime}}\right)+\frac{V V^{\prime}}{m_{a^{\prime}}}\right)+\mathbf{v} \frac{V^{\prime}}{r}\left(\mathbf{r} \cdot \mathbf{v}_{a}\right)\right\} \\
& \left.+\frac{1}{c^{3}} \epsilon \frac{2 e_{1} e_{2}}{3 m_{a^{\prime}}}\left\{\mathbf{r} \frac{2 s}{r}\left(\frac{V^{\prime}}{r}\right)^{\prime}+\frac{V^{\prime}}{r} \mathbf{v}\right\}\right]+O\left(\frac{1}{c^{4}}\right),
\end{aligned}
$$

where $\mathbf{r}=\mathbf{x}_{1}-\mathbf{x}_{2}, \mathbf{v}=\mathbf{v}_{1}-\mathbf{v}_{2}$, and $V(r)=e_{1} e_{2} / r$ is the Coulomb potential energy.

The accelerations $\mu_{a}(\mathbf{x}, \mathbf{v})$ given by (12) must now be compared with those that one derives from the Lagrangian (9). Expanding the Euler-Lagrange equations in a $1 / c$ series and taking Eq. (6) into account, we obtain from the $1 / c^{n}$ term in the expansion that

$$
\begin{aligned}
m_{a} \mu_{a}^{i(n)}= & \frac{\partial L^{(n)}}{\partial x_{a}^{i}}-\sum_{b=1}^{2} \\
& \times\left(v_{b}^{j} \frac{\partial^{2} L^{(n)}}{\partial x_{b}^{j} \partial v_{a}^{i}}+\sum_{m=0}^{n-1} \mu_{b}^{j(m)} \frac{\partial^{2} L^{(n-m)}}{\partial v_{b}^{j} \partial v_{a}^{i}}\right) .
\end{aligned}
$$

As is well known, for $n \leqslant 2$, the equations of motion (12) can be derived from the Darwin Lagrangian ${ }^{8}$

$$
L_{\mathrm{DW}}=L^{(0)}+\left(1 / c^{2}\right) L^{(2)}+O\left(1 / c^{3}\right) .
$$

(Notice that $L^{(1)}=0$.) A short calculation proves that the above equation is fitted by the Lagrangian (9) for

$$
f=-\left(e_{1} e_{2} / 4\right)\left(v^{2} / r\right)-\left(e_{1} e_{2} / 2 r^{3}\right) s^{2}, \quad \alpha=0 .
$$

For $n=3$, we have

$$
m_{a} \mu_{a}^{i(3)}=\frac{\partial L^{(3)}}{\partial x_{a}^{i}}-\sum_{b=1}^{2}\left(v_{b}^{j} \frac{\partial^{2} L^{(3)}}{\partial x_{b}^{j} \partial v_{a}^{i}}+\mu_{b}^{j(0)} \frac{\partial^{2} L^{(3)}}{\partial v_{b}^{j} \partial v_{a}^{i}}\right),
$$

which using (9), yields 


$$
\begin{aligned}
& m_{a} \boldsymbol{\mu}_{a}^{(3)} \\
& =\mathbf{r}\left[-\left(\frac{\gamma^{\prime}}{r}\right)^{\prime} \frac{s^{2} \eta_{a}}{r}-\frac{\gamma^{\prime}}{r}\left(\frac{y}{4}+\frac{1}{2} \frac{e_{1} e_{2}}{r} \frac{\eta_{a}}{m_{a}}\right)\right. \\
& -\gamma \frac{1}{2} \frac{e_{1} e_{2}}{r^{3}} \frac{\eta_{a}}{m_{a}}-g_{r s} \cdot \frac{s}{r}-g_{s s} \cdot \frac{1}{4}\left(y+\frac{e_{1} e_{2}}{r} \frac{1}{\mu}\right) \\
& \left.-g_{s y} \frac{2 e_{1} e_{2}}{r^{3}} \frac{s}{\mu}-g_{y} \frac{2 e_{1} e_{2}}{\mu r^{3}}+\frac{g_{r}}{r}\right]-\mathrm{v}\left[\frac{\gamma^{\prime}}{r} s \eta_{a}+g_{r y}\right. \\
& \left.\cdot \frac{4 s}{r}+g_{y y} \cdot \frac{8 e_{1} e_{2}}{r^{3}} \frac{s}{\mu}+g_{s y}\left(y+\frac{e_{1} e_{2}}{\mu r}\right)\right],
\end{aligned}
$$

where $g_{r}=\partial g / \partial r, g_{r s}=\partial^{2} g / \partial r \partial s$, etc., and the new variable $y=v^{2}$ has been introduced. Moreover, $\mu=m_{1} m_{2} /\left(m_{1}+m_{2}\right)$ stands for the reduced mass.

Then comparing (15) with the $1 / c^{3}$ term in the rhs of Eq. (12) and after some manipulation we arrive at

$$
\gamma(r)=0, \quad m_{1}=m_{2} \text {. }
$$

Consequently, the retarded (resp., advanced) electrodynamics of two-point charges does not admit a Lagrangian description approximated up to $1 / c^{3}$ unless both particles have the same mass.

In that case, the comparison of Eqs. (12) and (15) also yields the further condition

$$
\frac{\partial g}{\partial \mathbf{r}}-D\left(\frac{\partial g}{\partial \mathbf{v}}\right)=\frac{\epsilon e_{1}^{2} e_{2}^{2}}{3 \mu}\left[\frac{6 s}{r^{5}} \mathbf{r}-\frac{1}{r^{3}} \mathbf{v}\right],
$$

with

$$
D \equiv \mathbf{v} \frac{\partial}{\partial \mathbf{r}}+\frac{e_{1} e_{2}}{\mu} \frac{\mathbf{r}}{r^{3}} \frac{\partial}{\partial \mathbf{v}} .
$$
r),

Equation (17) splits into two scalar equations: (along

$$
\frac{2 g_{r}}{r}-\frac{1}{2} h_{s}=\frac{3 e_{1}^{2} e_{2}^{2}}{\mu} \frac{2 s}{r^{5}} ;
$$

(along v),

$$
g_{s}-2 h_{y}=-\epsilon\left(e_{1} e_{2} / 3 \mu r^{3}\right) ;
$$

where

$$
\begin{aligned}
h=D g & =g_{r} \frac{2 s}{r}+g_{s} \frac{1}{2}\left(v^{2}+\frac{e_{1} e_{2}}{\mu r}\right) \\
& =g_{y} \frac{4 s e_{1} e_{2}}{\mu r^{3}} .
\end{aligned}
$$

We stop at this point because whether or not Eqs. (19a) and (19b) are integrable has very little significance; indeed, they will be relevant only in the very special case of equal masses.

\section{CONCLUSION AND OUTLOOK}

We have proven that under rather unrestrictive conditions (invariance under space-time translations and rotations) there is no Lagrangian up to $1 / c^{3}$ for the retarded (resp., advanced) predictive electrodynamics of two-point charges with different masses; therefore, the no-interaction theorem for $1 / c$ expansions ${ }^{12}$ applies already at this order $\left(1 / c^{3}\right)$ for these theories. Nevertheless, it seems that this negative result only occurs for different masses.

As is usually done in relativistic theories of directly interacting particles, a possible way out would consist in dropping the condition that the configuration space is spanned by the particle positions $\mathbf{x}_{a}$, where $a=1,2$, and introducing a new set of configuration space coordinates $\mathbf{q}_{a}$ related to the former ones by

$$
\mathbf{x}_{a}=\mathbf{q}_{a}+\left[\left(m_{1}-m_{2}\right) / c^{3}\right] f_{a}(\mathbf{q}, \dot{\mathbf{q}}),
$$

where $f_{a}$ is a set of suitably chosen functions.

\section{ACKNOWLEDGMENT}

This work has been partially supported by CAICyT under Contract No. 0649-84.

${ }^{1}$ D. G. Currie, T. F. Jordan, and E. C. Sudarshan, Rev. Mod. Phys. 35, 350 (1963).

${ }^{2}$ H. Van Dam and E. P. Wigner, Phys. Rev. 142, 838 (1966); H. Leutwyler, Nuovo Cimento 37, 556 (1965).

${ }^{3}$ From the Hamiltonian point of view, see, R. N. Hill, J. Math. Phys. 8, 1756 (1967); L. Bel, Ann. Inst. H. Poincaré XIV, 189 (1971). From the Lagrangian point of view, see, E. H. Kerner, J. Math. Phys. 9, 222 (1968); J. Martin and J. L. Sanz, ibid. 19, 780 ( 1978); R. P. Gaida, Y. B. Klyuchkouskii and V. I. Tretyak, Teor. Mat. Fiz. 44, 194 (1980).

${ }^{4}$ P. A. M. Dirac, Rev. Mod. Phys. 21, 392 (1949).

${ }^{5}$ X. Jaen, A. Molina, and V. Iranzo, J. Math. Phys. 27, 512 (1986).

${ }^{6}$ X. Jaen, J. Llosa, and F. Marques, J. Math. Phys. 27, 519 (1986).

${ }^{7}$ S. de Bievre, J. Math. Phys. 27, 7 (1986).

${ }^{8}$ C. G. Darwin, Philos. Mag. 39, 537 (1920).

${ }^{9}$ A. Einstein, L. Infeld, and B. Hoffman, Ann. Math. 39, 65 (1938).

${ }^{10}$ F. Bopp, Ann. Phys. 38, 345 (1940); 42, 573 (1943).

"G. Breit, Phys. Rev. 34, 553 (1929); 36, 383 (1930); 39, 616 (1932).

${ }^{12}$ J. Martin and J. L. Sanz, J. Math. Phys. 19, 780 (1978).

${ }^{13}$ J. Martin and J. L. Sanz, J. Math. Phys. 20, 25 (1979).

${ }^{14} \mathrm{~V}$. N. Golubenkov and A. I. Smorodinski, Zh. Eksp. Teor. Fiz. 4, 442 (1957) [Sov. Phys. JETP 31, 330 (1958)].

${ }^{15}$ A. D. Fokker, Physica 58, 386 (1929).

${ }^{16}$ E. H. Kerner, J. Math. Phys. 3, 35 (1962).

${ }^{17}$ R. P. Gaida and V. I. Tetriak, Acta Phys. Pol. B 11, 509 (1980).

${ }^{18}$ L. Bel, A. Salas, and J. M. Sanchez Ron, Phys. Rev. D 7, 1099 (1973); R. Lapiedra and A. Molina, J. Math. Phys. 20, 1308 (1979).

${ }^{19}$ D. G. Currie, Phys. Rev. 142, 817 (1966); R. N. Hill, J. Math. Phys. 8, 201 (1967).

${ }^{20}$ L. Bel, Ann. Inst. H. Poincaré XII, 307 (1970). 